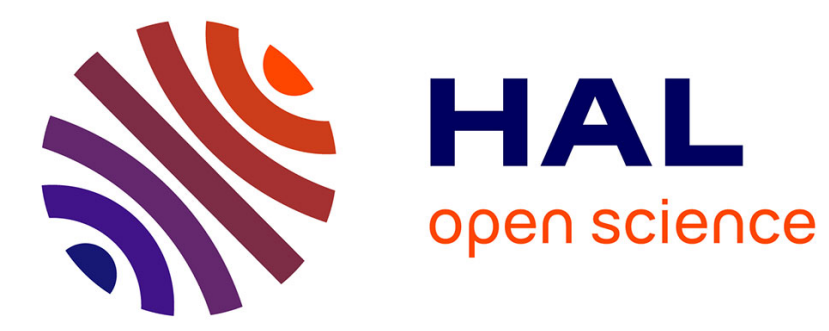

\title{
L'émotion et l'embargo, ou les deux corps d'Eliancito
} Ariel Colonomos

\section{To cite this version:}

Ariel Colonomos. L'émotion et l'embargo, ou les deux corps d'Eliancito. Critique Internationale, 2000, 8, pp.21-27. 10.3406/criti.2000.1435 . hal-01011343

\section{HAL Id: hal-01011343 \\ https://hal-sciencespo.archives-ouvertes.fr/hal-01011343}

Submitted on 23 Jun 2014

HAL is a multi-disciplinary open access archive for the deposit and dissemination of scientific research documents, whether they are published or not. The documents may come from teaching and research institutions in France or abroad, or from public or private research centers.
L'archive ouverte pluridisciplinaire HAL, est destinée au dépôt et à la diffusion de documents scientifiques de niveau recherche, publiés ou non, émanant des établissements d'enseignement et de recherche français ou étrangers, des laboratoires publics ou privés.

\section{(a)(1) $\$$}

Distributed under a Creative Commons Attribution - NonCommercial - NoDerivatives| 4.0 


\section{L'émotion}

Contre-jour

\section{et l'embargo, \\ ou les deux corps \\ d'Eliancito}

par Ariel Colonomos

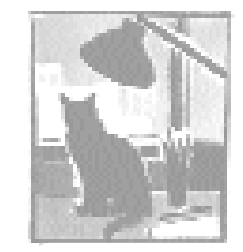

Contre-jour

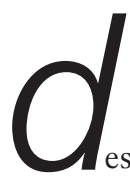

ois durant, l'histoire du petit Elian a tenu l'Amérique en haleine et a soulevé les passions à Cuba. Ce Cubain de six ans, repêché au large de la Floride le 25 novembre 1999 alors que sa mère avait trouvé la mort en tentant de gagner les États-Unis avec lui, est très vite devenu le centre d'une attention mondialisée. L'affaire est révélatrice des enjeux de l'émigration cubaine vers les États-Unis. Mais surtout, Elian sauvé des eaux est une métaphore vivante de notre monde post-bipolaire. Sa tragédie témoigne de la place des émotions dans un monde d'images sans frontières. À la faveur d'une théâtralité nouvelle, la diffusion des sentiments polarise les consciences et les identités, elle scinde aussi les corps. Enfin, cette irruption de l'« humain » dans le jeu des États discrédite l'embargo obsolète qui régit encore les relations entre les deux pays concernés.

\section{L'enjeu migratoire}

L'émigration cubaine vers les États-Unis est traditionnellement au centre des litiges et des négociations entre les deux voisins. Si les émigrés de 1959, lors de la Révolution, avaient été bien accueillis, de nombreux opposants au castrisme trouvant alors refuge dans de bonnes conditions au sein du système américain, les vagues qui ont suivi ont connu un sort moins heureux. La perspective d'un nouvel afflux - à l'image de celui du début des années quatre-vingt ${ }^{1}$ - effraie. Face à l'arrivée de ces désenchantés du communisme tropical ou de ces enthousiastes du rêve américain, les réponses sont maladroites, à très courte vue, et peu adaptées aux normes humanitaires, ainsi qu'aux exigences des familles cubaines de Miami soucieuses d'accueillir leurs proches.

En 1994, une nouvelle vague de Cubains échoue sur les côtes de la Floride : ce sont plus de 30000 personnes qui fuient une situation économique brutalement aggravée par la perte du soutien soviétique et le durcissement de l'embargo américain (loi Torricelli de 1992). Clinton et Castro signent alors un accord de contrôle 
migratoire, dans lequel les États-Unis s'engagent à accueillir chaque année 20000 Cubains sur la terre promise ${ }^{2}$. Ces négociations ont montré l'habileté du lider maximo, qui a su faire de l'émigration - embarrassante en tant que désaveu de la politique révolutionnaire - un instrument de gestion de son pouvoir : en menaçant d'ouvrir les vannes migratoires, il renforce sa position face aux États-Unis. Quoi qu'il en soit, l'accord n'apporte aucune satisfaction aux Cubains désireux d'émigrer ni à leurs familles de Miami : d'une part le quota de visas est insuffisant par rapport au nombre de candidats au départ, potentiels ou déclarés, d'autre part le refoulement, par les garde-côtes américains, des irréguliers qui tentent l'aventure sur des radeaux (balseros) n'est pas encourageant. Les conditions sont réunies pour faire du tragique destin d'Elian un emblème politique.

En face, s'appuyant sur une population attendrie par le sort de l'enfant, Fidel dénonce le complot dont Cuba est encore une fois victime : on prétend arracher un enfant à son pays. Ce fossile de la guerre froide a rapidement saisi la visibilité mondiale qu'un tel fait divers assure au nouveau défi qu'il jette aux États-Unis. Il compose son rôle de patriarche hérö̈que en usant avec brio des ressorts de la communication post-bipolaire ${ }^{3}$ et proclame vigoureusement ses droits sur l'enfant. Paternité, paternalisme et populisme œuvrent de concert.

\section{Confusion des sentiments et jeux d'autorité}

Les moindres faits et gestes de ce Moïse latino ont été scrutés par les Américains depuis le jour de son repêchage miraculeux. Sa vie a été suivie jusqu'au plus intime par les télévisions et les journaux du pays. Les Européens n'ont pas été épargnés par cette singulière combinaison de compassion et de voyeurisme. Deux dimensions a priori disjointes se sont associées : d'une part l'évaluation des conditions objectives d'existence d'un jeune enfant dans un des derniers bastions du communisme, de l'autre une plongée dans les ultimes recoins de son for intérieur. Deux domaines sont constamment imbriqués : le public et le privé, le public le plus vaste, dans sa dimension mondiale, et le privé le plus secret et le plus intime, le registre subjectif d'une jeune conscience.

Le débat public a impliqué un constant va-et-vient entre Cuba, la Floride et Washington, les rigueurs spartiates du régime castriste, les rues bruyantes de Little Havana à Miami et les couloirs du pouvoir, au Congrès et à la Maison Blanche. Deux États antagoniques depuis quarante ans ont poussé leurs plus hauts représentants sur le devant de la scène pour s'exprimer sur l'affaire. Et l'embargo sévère qui régit leurs relations a été de plus en plus critiqué.

La scène privée n'est pas moins bien documentée. Chaque jour inlassablement, les moindres paroles de l'enfant ont été traquées par les micros, ses sourires photographiés, ses modestes vœux matériels exaucés. Sous la loi de la concurrence, les télévi- 
sions se sont âprement disputé chaque image. L'un des points culminants de cette exhibition a été, en avril, lors de l'arrivée du père à Washington, l'épisode où le petit Elian manifeste son désir de rester aux États-Unis. Les médias sont indéniablement responsables des échos publics de cette voix intime, et de ses résonances politiques.

À peine repêché, l'orphelin - son père est encore absent de la scène - devient l'étendard des lobbies anti-castristes de Miami. La médiatisation à outrance de son histoire impose de leur part une réponse vigoureuse : rester hors de cette arène, c'est perdre pied au sein d'une communauté dont les membres s'identifient massivement à la figure du garçonnet. Les différents mouvements, tels que la célèbre Cuban American National Foundation, assignent l'enfant à résidence auprès de sa nouvelle famille, son grand-oncle et tous ceux qui lui prodiguent des cadeaux destinés à lui faire oublier ses malheurs et les rigueurs de l'île. Il n'est pas de semaine sans que de bruyantes manifestations ne soient organisées, aussi bien en Floride qu'à Washington ou à New York, par ces survivants de la guerre froide, qui réussissent depuis déjà dix ans à résister à l'oubli. Ces groupes avaient fait leur entrée dans la politique américaine sous les auspices de Reagan, qui voyait là un accès direct à un précieux réservoir de voix. Au cours des années quatre-vingt, leur structure de représentation des intérêts a été directement calquée sur le modèle des lobbies juifs pro-israéliens. Très rapidement, les diverses fondations, ainsi que Radio et TV Marti, ont drainé des fonds considérables, qui ont contribué au financement des deux grands partis ${ }^{4}$. Dans l'affaire d'Elian, le message de ces mouvements ne s'embarrasse pas de nuances : rendre l'enfant à Cuba, c'est « renvoyer un petit juif en Allemagne en $1940 »$.

Pour les responsables de la politique étrangère américaine, cette malheureuse histoire souligne - une fois de plus - la nécessité d'un règlement de l'embargo. Une bienveillante neutralité diplomatique est alors de mise. Les signes officiels d'une volonté d'apaisement sont manifestes, et le Service de l'immigration et de la naturalisation (INS) se prononce en faveur du retour de l'enfant. Cet avis va à l'encontre du jugement rendu en Floride par la juge Rosa Rodriguez, qui se heurte également à la décision fédérale de régler l'affaire à l'échelle nationale. Le décalage entre Washington - la Présidence, le Département d'État, le ministère de la Justice, les différents think tanks, les intellectuels - et les notables de la scène cubaine de Floride ainsi que leurs relais au Congrès ne cesse de grandir.

En phase avec le sentiment de bon nombre de citoyens américains lassés par les effets de l'embargo vis-à-vis de Cuba, Clinton et le Département d'État poursuivent leur politique de «people-to-people contacts», au regard de laquelle garder Elian en Floride contre l'avis de son père cubain serait à proprement parler une hérésie. La volonté de rétablir des rapports culturels entre les peuples, en construisant des liens entre les deux sociétés civiles notamment par la diplomatie du sport - base-ball ou football - trouve un prolongement tout naturel avec l'entrée en scène de l'enfance. 
Enfant symbole, enfants diplomates : au cours de l'hiver, des élèves d'une école de l'Oregon rendent visite à leurs « homologues » de La Havane et ces bons petits, ayant débattu de l'embargo, votent sa levée par 49 voix contre 3 dans l'enceinte d'une école Lénine de la banlieue de la capitale. Les jeunes Cubains réclament bien évidemment le retour d'un des leurs. De nombreux Américains le souhaitent également, Elian doit retrouver son père. Forts de ce soutien dans l'opinion, les cercles les plus officiels veulent accélérer ce retour : la séparation d'avec son père va contre le bon sens et les règles policées d'une civilisation heureuse qui célèbre les vertus de la famille. L'humain avant tout. L'État se prononce en faveur du père biologique.

C'est compter sans les intérêts électoraux. Les Cubains américains font valoir le droit du père symbolique : Elian est le fils d'une communauté qui transcende la simple filiation biologique. Or il y a là une importante réserve de voix. Face à la volonté du Département d'État et de Clinton de régler l'affaire en rendant le garçon à son père, les hommes politiques américains directement concernés par les prochaines élections prêtent l'oreille aux Cubains américains. Al Gore, viceprésident et candidat démocrate pressenti, n'hésite pas à aller au-devant de leurs exigences (il l'avait déjà fait l'année dernière en demandant au Président de ne pas créer de commission bi-partisane sur l'embargo, qui aurait sans doute recommandé sa révision). Si le candidat républicain George $W$. Bush déclarait dès avant l'affaire ne pas vouloir toucher à l'embargo une fois élu à la présidence, Al Gore, lui, va se prononcer pour l'octroi rapide de la citoyenneté américaine au rescapé : bref, on songe à modifier les lois de l'Amérique pour s'octroyer ce corps, pour le garder en tant que précieux symbole de l'accès au monde libre.

L'affaire met aux prises différentes figures de l'autorité. D'un côté, les autorités politiques fédérales, considérant que l'enfant a un tuteur légal en la personne de son père, veulent le rendre à ce dernier. La police entre en force au domicile du grand-oncle, le 22 avril, et s'empare de l'enfant. De l'autre, Elian est revendiqué et « adopté » par la communauté des Cubains américains de Miami. Celle-ci s'est constituée en tant que père de substitution et assume la charge de la paternité sur un plan symbolique. Il existe dès lors un deuxième corps d'Elian, un corps sacré et symbolique qui suscite émotion et ferveur tout en déchaînant les passions politiques. Cette division des corps est rendue possible par le chevauchement des autorités auquel le monde de l'après-guerre froide nous a désormais accoutumés. L'autorité ne s'incarne pas dans une justice unique et universellement reconnue, et les mobilisations identitaires, tout comme la prolifération des acteurs non étatiques, nous rappellent sans cesse que la mondialisation des images a pour conséquence de redéfinir les lieux et les supports de l'identification des individus 5 .

L'autorité incarnée par la communauté des Cubains américains met en scène ce deuxième corps ${ }^{6} \mathrm{~d}^{\prime} E$ lian. Les médias deviennent rapidement le théâtre de cette représentation identitaire. Elian est un héros, c'est ce rôle qui a été assigné à son 
corps. Martyr s'il rentre sur l'île, héros de la liberté acquise au prix de la vie de sa mère s'il reste en Amérique. En raison de son destin tragique, de son inscription dans le registre du miracle, le rescapé acquiert une dimension religieuse, ce dont témoigne toute l'iconographie christique qui accompagne les manifestations dans les rues de Miami en faveur de son installation en Floride. Tel Jésus, l'enfant n'a plus de père biologique, son ascendance est désincarnée. La nature exceptionnelle de cette destinée ne peut échapper à une communauté en quête d'emblèmes forts depuis que la menace géostratégique du castrisme a disparu. Accaparer l'enfant, c'est s'identifier à la fois à la souffrance, à la justice et à l'espoir. Les passions internationales sont d'autant plus vives que les enjeux sécuritaires sont moins déterminants.

Curieusement, le principe d'un deuxième corps sacré d'Elian est validé par le pouvoir castriste. Pour celui-ci comme pour de nombreux habitants de l'île - en témoignent les rassemblements -, le garçon a bel et bien été « kidnappé » à Miami. Le régime met en accusation les fanatiques de l'embargo, ces guzanos ${ }^{7}$ de Floride responsables d'un crime qui touche de nouveau les plus faibles, les dominés chez les dominés, l'enfance malheureuse du Sud. On peut désormais imputer à ces « tueurs d'enfants $\gg^{8}$ un nouveau crime, la séquestration d'un orphelin loin de son père. Alors que les dirigeants des groupes anti-castristes proclament qu'Elian est leur « fils », La Havane en appelle à la médecine, à la psychiatrie et à la psychologie - en renfort du droit international - pour dénoncer les méfaits d'une telle usurpation de la paternité. Le corps sacré d'Elian est tiraillé entre les deux rives. Le régime de La Havane et les anti-castristes de Miami concourent ainsi tous deux à rendre plus difficile l'exercice de la diplomatie.

Cette affaire n'est pas sans rappeler une autre série de péripéties d'une époque révolue ${ }^{9}$. En 1858, à Bologne, un enfant juif du même âge qu'Elian est soustrait à ses parents par l'autorité pontificale après avoir été « secrètement » converti au catholicisme par sa nourrice. Cette affaire va mobiliser pendant douze ans l'Europe des chancelleries et de la presse, déclenchant une véritable campagne internationale de protestation en faveur de la restitution du jeune Edgardo Mortara à ses parents. Les démarches de ces derniers ainsi que les tentatives politiques de règlement, au surplus ralenties en raison de la guerre d'Italie, restent longtemps vaines. Après l'instauration d'un gouvernement républicain, le pouvoir pontifical ne peut plus retenir le jeune homme à Rome. Douze années après son enlèvement, Edgardo Mortara retrouve donc ses parents, qui constatent amèrement son refus de quitter la religion qu'il avait été contraint d'embrasser : rendu à sa liberté, il décide d'entrer dans les ordres et devient moine. Il s'éteindra dans une abbaye belge en 1940...

Cette histoire témoigne de la particularité des trajectoires individuelles lorsqu'elles croisent l'histoire des relations entre les États. Les démarches compliquées des parents Mortara ne sont pas sans rappeler les tribulations du père d'Elian. Et les Cubains américains de Miami n'ont cessé de clamer que l'enfant ne voulait pas retourner sur 
l'île. Si, en d'autres temps, la volonté d'Edgardo n'a été connue qu'à sa majorité, l'espace médiatique d'aujourd'hui est d'emblée à l'affût du désir de ce petit garçon violemment propulsé « dans la cour des grands ». Son corps repêché, puis ses paroles de miraculé sont autant de nouveaux paramètres de la scène internationale. Fautil voir dans cette extraordinaire mise en scène internationale de l'intime une victoire du respect de la personne humaine ? Entre le désir défunt d'une mère noyée et la parole publique d'un père vivant sous haute surveillance, que choisir?

\section{La mise en cause de l'embargo}

La médiatisation de cette histoire politico-familiale a fait tomber la frontière entre public et privé : l'embargo se voit critiqué en raison même de l'attention portée aux souffrances de l'enfant. Si les interdits commerciaux existent depuis 1960, ils n'ont vraiment frappé au cœur qu'en 1989, par suite de l'implosion de l'URSS : Cuba, soudain complètement isolé, traversera alors une phase difficile, jusqu'au moment où les effets de sa coopération commerciale avec l'Europe, le Canada et le Mexique se feront sentir. Mais l'embargo a encore été renforcé deux fois : en 1992 (loi Torricelli) et en 1996 (loi Helms-Burton). La loi de 1992 empêche les filiales américaines à l'étranger de commercer avec l'île. Celle de 1996 prévoit des mesures de rétorsion contre les firmes non américaines qui traitent avec des unités cubaines provenant de la nationalisation de biens ayant appartenu à des Cubains américains ${ }^{10}$. En janvier 1999, Clinton assouplit l'embargo autant que le lui permet la loi - c'est-à-dire très peu ${ }^{11}$. L'affaire d'Elian s'inscrit dans le contexte de l'après Helms-Burton, au moment où la société civile américaine - entreprises et mouvements humanitaires - se montre de plus en plus critique vis-à-vis de l'embargo et de ses conditions d'application.

Dès lors, l'espace international se subjectivise. À mesure que la crise prend de l'ampleur, la politique américaine vis-à-vis de Cuba est soumise à une évaluation de plus en plus critique. Les médias ne parlent pas que des larmes de l'enfant. Ils offrent un espace de parole aux adversaires de l'embargo et aux mouvements humanitaires ou religieux. Les Églises proposent leurs services. L'embargo serait d'autant plus injuste qu'il a conduit à ce drame horrible, et le dilemme doit être résolu par le retour de l'enfant. C'est un coup très dur pour les sanctions, et c'est sans doute une des raisons de la ferveur rageuse et maladroite des anti-castristes de Floride durant toute l'affaire. La tragédie d'Elian est mise au passif d'une loi inutile - et même contre-productive, car elle fournit une occasion de légitimation au régime castriste. Elle montre les méfaits du trop grand espace occupé par les dinosaures de l'anticommunisme à Miami.

Plusieurs points sont rappelés à l'occasion des nombreux débats que ces mois de suspense ont suscités. De manière générale, les sanctions unilatérales sont faiblement 
légitimes. Lorsque les embargos sont commerciaux, ils ne doivent pas toucher des domaines où la vie humaine est en jeu, les secteurs agro-alimentaire et médical. Or une telle restriction continue à faire partie de l'embargo vis-à-vis de $\mathrm{Cuba}^{12}$. En définitive, l'histoire d'Elian ne fait que renforcer le sentiment de lassitude éprouvé aux États-Unis à l'égard de cette politique. Les Cubains américains sont seuls à la défendre, secondés par des républicains de moins en moins nombreux et une poignée de démocrates directement concernés par la Floride. La passion suscitée par l'affaire nous rappelle que les lois des États tiennent aussi à des paramètres proprement humains. La stratégie adoptée par le président Clinton le montre bien : le Département d'État, explique-t-il, aurait intérêt à faire montre d'humanité et à prendre ses distances avec la ligne de conduite imposée par le Congrès ces dernières années. La honte et la culpabilité entravent la politique d'un pays, et mieux vaut être à l'origine de sentiments favorables qu'avoir à subir la désapprobation ou l'humiliation. Aujourd'hui, les Américains sont confrontés à un double écueil. Impuissants, ils sont responsables de l'inefficacité de leur politique et du maintien d'un des plus grands défis symboliques à leur hégémonie. Cruels, ils doivent assumer la charge de leur sadisme réfracté dans les larmes d'un enfant. Peut-être les résultats des prochaines élections législatives ouvriront-ils enfin la voie à une sortie des sanctions.

Elian est à l'image du monde d'aujourd'hui : les identités se publicisent tandis que la « grande politique »- la politique internationale - se privatise, et le monde devient le théâtre de ces représentations nouvelles. Un monde que n'arrête aucune frontière, pas même celles de l'intériorité.

\footnotetext{
1. En 1980, 125000 Cubains ont quitté l'île par le port de Mariel.

2. Ce quota était déjà mentionné dans des accords de 1984, mais le nombre réel de visas accordés a toujours été bien inférieur. 3. Pour une étude du statut et du rôle des émotions et de la passion dans les relations internationales, voir Neta C. Crawford, «The passions of world politics. Propositions on emotion and emotional relationships », International Security 24 (4), printemps 2000, pp. 116-156.

4. Patrick Kiger, Squeeze play. The US, Cuba and the Helms-Burton Act, Washington, The Center for Public Integrity, 1997. 5. James Rosenau a été un des premiers auteurs à souligner ce phénomène international, la redéfinition de l'autorité. James Rosenau, «The redefinition of authority in a shrinking world », Comparative Politics 24 (3), avril 1992, pp. 253-273.

6. Pour paraphraser Kantorowicz, l'affaire d'Elian c'est les deux corps du Prince. Chacun se presse pour exercer son droit de paternité sur un corps aussi prestigieux. Au corps réel juridiquement lié à son père biologique, les Cubains américains opposent un corps symbolique, un emblème qu'ils tentent de se réapproprier. Malgré le départ physique d'Elian de Miami, l'effigie demeure. Voir Ernst Kantorowicz, Les deux corps du roi, Paris, Gallimard, 1957.

7. «Vers de terre », surnom dont les Cubains qui ont émigré aux États-Unis sont affublés par le régime et ses sympathisants. 8. Accusation déjà ancienne des castristes à l'encontre des partisans intransigeants de l'embargo, auquel seraient attribuables les difficultés sanitaires de Cuba.

9. Mes remerciements à Christophe Reffait et Joëlle Menrath, historiens du XIXe siècle, pour avoir attiré mon attention sur l'« affaire Mortara ». David I. Kertzer, The Kidnapping of Edgardo Mortara. New York, Vintage Books, 1998.

10. Ces mesures n'ont cependant pas été appliquées à l'encontre des firmes européennes, alors qu'elles étaient les premières visées. 11. Voir http://www.state.gov/www/regions/wha/cuba/policy.html

12. À la faveur de l'affaire Elian, cet aspect du débat a été relancé au Congrès, et un projet de loi levant cette restriction a été approuvé par le Sénat.
} 